\title{
Comparison of latching control strategies for a heaving wave energy device in random sea
}

\author{
A. Babarit, G. Duclos, A.H. Clément ${ }^{\star}$ \\ Laboratoire de Mécanique des Fluides (CNRS UMR6598), Ecole Centrale de Nantes, 1 Rue de la Noë, BP 9210144321 Nantes cedex 3, France
}

\begin{abstract}
This paper investigates semi-analytically the latching control applied to a mechanical oscillator; and numerically three strategies of latching control for a point absorber wave energy converter oscillating in the heave mode only. By solving the equation of motion of a mechanical damped oscillator, it is shown that latching control can magnify the amplitude of the motion whatever the frequency of the excitation force, and how it can improve the efficiency of the system, in term of absorbed energy, for excitation frequencies apart from the natural frequency. Assuming that the excitation force is known in the close future and that the body is locked in position at the current time step, equations of motion of the body are solved numerically in the time domain for different initial conditions (i.e. latching durations). For all these simulations, three criteria_one for each strategy — are tested and the latching time leading to the best result is selected. Time domain simulation results are presented for a heaving buoy in small-amplitude regular and random waves. In regular waves, the same results as for the case of a mechanical oscillator are recovered for the wave energy converter. In random sea, results show that for all the three proposed strategies, efficiency of the wave energy converter is considerably improved in terms of absorbed energy. Numerical study of the period of the controlled system shows that the delay of prediction of the excitation force in the future seems to be bounded by the natural period of the system.
\end{abstract}

Keywords: Wave energy; Point absorber; Latching control; Random sea

\section{Introduction}

The aim of the study was to assess the benefit brought to a simple point absorber wave energy device by a discrete latching control, in random waves conditions. A generic and ideal wave energy device is considered: a vertical circular cylinder being free to move in a single vertical mode of motion, all five other motions being restrained by an ideal frictionless mechanism (Fig. 1). In calm water, it remains at a fully submerged equilibrium position under the action of its weight, its buoyancy and a linear spring force (stiffness $k$ ). The Power Take Off (PTO) mechanism is idealized here by a linear damping coefficient $B$, giving a force proportional to the vertical velocity of the cylinder.

\footnotetext{
* Corresponding author. Tel.: +332403725 26; fax: +332403725 23.

E-mail address: alain.clement@ec-nantes.fr (A.H. Clément).
}

Hydrodynamic forces due to wave diffraction and radiation are modelled using linear water wave theory; they appear as a convolution product over the vertical velocity in the equations of motions, according to the standard Cummins' decomposition. The device is thus described as a linear, single DoF, mechanical oscillator; and the numerical model that we have used in the study is based on a linear modelling of the hydrodynamics. But applying a (highly) nonlinear mode of control like latching makes the whole process a nonlinear oscillator featuring parametric resonances, which may be exploited to enhance the energetic performance of the plant. The latching control of wave energy devices, proposed by Budal and Falnes in 1980 [3] consists of locking the oscillating body in position at the instant when its velocity vanishes, and releasing it after a certain delay to be determined, when the wave forces are in good phase to maximize a criterion over the next oscillation. The benefit of such a control may be assessed semi-analytically for simple systems in harmonic excitation (regular forces). For the simulation in random waves which was the final goal of the study, a time stepping numerical simulation has been 
implemented, based on linear water wave theory, considering the wave excitation to be known in the near future beyond the current time step. Three criteria on the body motion on the next ramp-what we called strategies-were defined to determine the optimal moment to release the body. A large number of random sea states, based on discretized PM spectrum with varying peak period but constant characteristic height, were tested with a $10 \mathrm{~m}$ diameter cylindrical device tuned at $10 \mathrm{~s}$ natural period. The mean power absorbed by the PTO was computed over long simulation periods, and then was expressed in terms of capture width, as usual when studying wave energy devices.

\section{General formulation}

In this study, we shall focus on the control of a generic point absorber device with a single degree of freedom (DoF). Namely, we will consider a submerged vertical cylinder constrained to move in heave motion only, under the action of wave excitation forces but all the theoretical work presented here can be applied to the more common case of floating bodies (provided the linearized buoyancy force is included in the spring force).

\subsection{Free motion}

The body oscillates vertically under the action of: excitation forces, radiation forces, restoring forces idealized

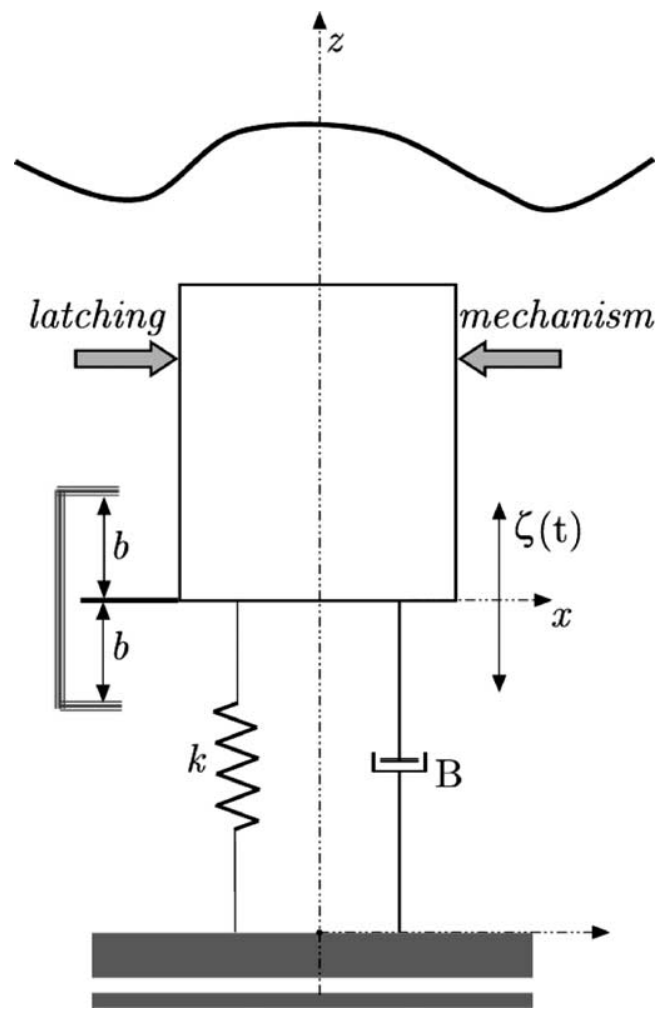

Fig. 1. A generic single DoF wave energy device. here as a single spring of stiffness $k$, and a damping force proportional to the velocity (with damping coefficient $B$ ) supposed to represent the action of the external Power Take Off (PTO) mechanism. The vertical motion around the equilibrium position will be denoted by $\zeta(t)$. This generic system belongs to the same family as the Archimedes Wave Swing (AWS) system, which has been tested in Portugal. A linear approach will be adopted here for modelling the hydrodynamics, in such a way that the behavior of the body in waves is governed by the following integro-differential equation

$\left(M+\mu_{\infty}\right) \ddot{\zeta}+\int_{0}^{t} \dot{\zeta} K(t-\tau) \mathrm{d} \tau+B \dot{\zeta}+k \dot{\zeta}=F_{\text {ex }}(t)$

which is the so called Cummins' decomposition [9] of the radiation forces into an instantaneous added mass term $\mu_{\infty} \ddot{\zeta}$ and a memory term expressed by a convolution product $\int_{0}^{t} \dot{\zeta}(\tau) K(t-\tau) \mathrm{d} \tau$. The kernel $K$ of this integral, generally named the impulse response function or sometimes the retardation function, depends essentially on the shape of the body. It can be computed directly in the time domain by using seakeeping dedicated BEM codes like ACHIL3D [8], TIMIT,.... It can also be deduced by a Fourier transform of the frequency domain hydrodynamic coefficients (added mass and damping coefficients) for which computer codes like WAMIT [1], DIODORE, AQUADYN [10],... can be used. In the present time-domain linear formulation, the free motion $\zeta(t)$ of the device may be calculated by integrating (1) for a given history of the wave excitation forcing term $F_{\text {ex }}(t)$, and given initial conditions $\zeta(0)$ and $\dot{\zeta}(0)$. Once the impulse response function $K$ has been determined, it can be further approximated by a sum of exponential functions by using the Prony's method as described in [11]. This allows replacing the integro-differential form (1) by a system of ordinary differential equations (ODE) like (25), generally better suited to numerical simulation. The device as described up to now is a linear mechanical oscillator. The mean power $\hat{P}$ extracted by the PTO over a time range $T$ is, therefore, given by:

$\hat{P}=\frac{1}{T} \int_{0}^{T} B \dot{\zeta}^{2}(\tau) \mathrm{d} \tau$

When the mechanical parameters $B$ and $k$ remain constant and no latching occur, the device is said to be uncontrolled.

\subsection{Latching control}

This device is basically a linear mechanical oscillator, with a natural circular frequency $\omega_{0}$ depending on the spring coefficient $k$, on the proper mass and the added mass of the body. When it is supposed to be left uncontrolled, the designer must tune the natural frequency to the local sea state statistics of the site where the device will be deployed. This is indeed the simplest but poorest way to optimize the power captured by the device. A further step in this 
uncontrolled configuration is to allow for different discrete values of the mechanical characteristics (say $B$ and $k$ here) in order to allow a certain adaptation of the oscillator to the variation of the sea state, but in an average sense. The device can therefore be adapted to the variation of the spectrum, after averaging over large period of time (say hours). A device is said to be controlled if the mechanical characteristics can be varied in order to adapt continuously the system to the incident wavetrain, wave after wave. The control can be either continuous or discrete (see Falnes [15] for a recent review of this topic).For an axisymmetrical wave-absorbing device in regular waves, it is known [14] that the maximum power that can be absorbed equals the incident wave power associated with a wave front of width one wavelength divided by $2 \pi$. This result was first derived independently by Budal and Falnes [2], Evans [13] and Newman [22]. Still according to Falnes [14], applying a control known as 'reactive control' to the device allows absorption of the maximum power. Actually, reactive control leads the motion of the body to fulfill two conditions. The first one states that the oscillating velocity of the body must be in phase with the excitation force on the body. This happens naturally when the wave frequency equals the natural frequency of the body, but one must act on the body when it is not the case in order to respect it. This is known as phase control. The second condition is called the optimum amplitude condition. Basically, if the amplitude is unconstrained, this condition is that the resistive load $B$ of the PTO must be equal to the hydrodynamic damping coefficient at the incident wave frequency. In case of optimum control, continuous control can be achieved by acting on the parameters of the PTO in order to respect these two conditions. Lots of work have been done about this topic, see e.g. [5,6,11]. In the present work, we have put the focus on another approach to Wave Energy Converters (WECs) control, called latching control. Unlike the previous one, this method is discrete. It consists in locking (latching) the motion of the body at the moment when its velocity vanishes, and waiting for the wave force having reached the most favorable phase to release the body (see Fig. 4). The body then starts moving from this initial position to the next vanishing velocity position where it is latched again, and so on,... Instead of being a smooth, continuous function, the position of the body is a succession of ramps of transient motions separated by stages of rest. The action upon the system is therefore binary: either the body is latched, or it is free to move, which explains the meaning of the term 'discrete' used in this context. The instant of latching is imposed by the dynamics of the body itself (i.e. vanishing velocity); thus, the control variable is simply the duration of the latching phase, or equivalently the instant of release. This mode of control, applied to the heave motion of the buoy, was proposed by Budal and Falnes [16,3] and further investigated experimentally by Budal et al. [4] in irregular waves and numerically in irregular waves by Iversen [20] and Eidsmoen [12] and in regular waves by Greenhow and
White [17] and Hals et al. [18]. It is shown in the last paper that one of the advantages of the latching control is that the ratio between the maximum absorbed power and the average absorbed power is smaller than the one of the reactive control. Obviously, this control cannot lead to an absolute respect of the optimality conditions, but it gives nevertheless very good results in terms of absorbed power. In the formerly quoted papers dealing with numerical simulations of latching control in irregular waves, the authors generally assumed that the excitation force was known sufficiently far in the future. We will make the same assumption in the present study. It is indeed a strong hypothesis, which will require some short-term forecasting process to implement the present latching strategies in real world. Such algorithms exist and they will be used in due time; such developments are beyond the scope of the present study which is simply aimed at assessing and comparing some latching strategies in random waves. However, note that Budal et al. [4] used a Kalman filter in order to predict the incident wave when applying latching control in the experiments reported in with success.

\section{Latching control of a simple mechanical oscillator under harmonic excitation}

The study of latching control of a simple mechanical oscillator consisting of a mass, a damper, and a spring under harmonic excitation will help us to understand the mechanical phenomena involved.

\subsection{Analytical calculation of the latching time}

The aim of the latching control will be here to maximize the amplitude of the oscillations of the mass. We will see that it will also maximize the absorbed power at the same time. When the excitation force is harmonic and when the system is a simple mechanical oscillator described by Eq. (3), one can solve analytically the problem of determining the optimal latching duration. Indeed, the motion of such a controlled system is composed alternately of:

- periods of rest, when the body is held. During these phases, the position of the body is constant, and its velocity equals 0 ,

- periods of transient motion, here called 'ramp', for which we can calculate analytically the motion in the case of a simple oscillator governed by the following differential equation

$$
\ddot{\zeta}+2 \mu \dot{\zeta}+\omega_{0}^{2} \zeta=F_{\text {ex }} \cos \left(\varphi_{0}+\omega t\right)
$$

where:

- $\omega_{0}$ is the natural frequency of the body.

- $\mu$ is the damping coefficient (essentially positive). 
- $F_{\mathrm{ex}}$ is the modulus of the excitation force.

- $\omega$ is the frequency of the excitation force.

- $\phi_{0}$ is the phase of the excitation force at the initial time $t=0$.

Let $\sigma=\sqrt{\omega_{0}^{2}-\mu^{2}}$, the general solution of Eq. (3) can be expressed in real notation by

$$
\begin{aligned}
\zeta(t)= & (a \cos (\sigma t)+b \sin (\sigma t)) \mathrm{e}^{-\mu t} \\
& +H(\omega) F_{\text {ex }} \cos \left(\varphi_{0}+\omega t+\varphi(\omega)\right)
\end{aligned}
$$

with

$\tan (\varphi(\omega))=\frac{-2 \mu \omega}{\left(\omega_{0}^{2}-\omega^{2}\right)}$

$H(\omega)=\frac{1}{\sqrt{\left(\omega_{0}^{2}-\omega^{2}\right)^{2}+(2 \mu \omega)^{2}}}$

and $(a, b)$ is a pair of real variables depending on the initial conditions.

\subsection{Ramps with alternated maxima}

We further suppose that the body will move during its transient motion from a position $\zeta_{0}$ to the position $-\zeta_{0}$, alternating the sign of the maxima of the successive ramps. It means that starting from an initial state $\zeta_{0}$ with the zero velocity, we want the body position to be equal to $-\zeta_{0}$ at the next stop. So, as summarized in Fig. $2:^{1}$

- The body is locked in the position $\zeta_{0}$ for $t \leq 0$. So $\dot{\zeta}(0)=0$.

- The initial phase of the excitation force is such that at time $t_{1}$, defined by $\dot{\zeta}\left(t_{1}\right)=0$, we have $\zeta\left(t_{1}\right)=-\zeta_{0}$.

So, from these considerations, we write the initial conditions as

$$
\left\{\begin{array}{l}
\zeta(0)=\zeta_{0} \\
\dot{\zeta}(0)=0
\end{array}\right.
$$

and the final conditions:

$$
\left\{\begin{array}{l}
\zeta\left(t_{1}\right)=-\zeta_{0} \\
\dot{\zeta}\left(t_{1}\right)=0
\end{array}\right.
$$

Using Eq. (4) with boundary conditions (7) and (8), and by combination in order to remove $a$ and $b$, we get the following system of equations

$$
\left\{\begin{array}{l}
A\left(t_{1}, \varphi_{0}\right) \zeta_{0}+B\left(t_{1}, \varphi_{0}\right) F_{\mathrm{ex}} H(\omega)=0 \\
C\left(t_{1}, \varphi_{0}\right) \zeta_{0}+D\left(t_{1}, \varphi_{0}\right) F_{\mathrm{ex}} H(\omega)=0
\end{array}\right.
$$

with:

\footnotetext{
${ }^{1}$ Files containing the data of all the figures of this paper are freely available at http://www.ec-nantes.fr/dhn/Francais/publi/public.html.
}

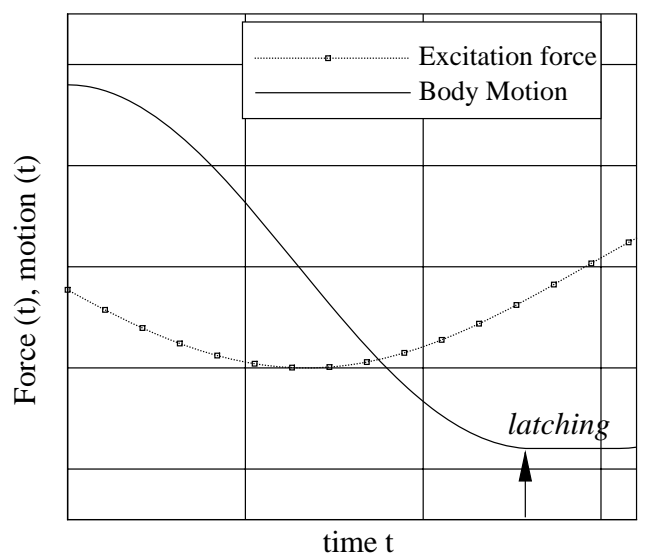

Fig. 2. A ramp of the body response.

$$
\begin{aligned}
A\left(t_{1}, \varphi_{0}\right)= & \left(\cos \sigma t_{1}+\frac{\mu}{\sigma} \sin \sigma t_{1}\right) \mathrm{e}^{-\mu t_{1}}+1 \\
B\left(t_{1}, \varphi_{0}\right)= & \left(-\cos \sigma t_{1} \cos \left(\varphi_{0}+\varphi(\omega)\right)+\frac{\sin \sigma t_{1}}{\sigma}\right. \\
& \left.\times\left(\omega \sin \left(\varphi_{0}+\varphi(\omega)\right)-\mu \cos \left(\varphi_{0}+\varphi(\omega)\right)\right)\right) \\
& \times \mathrm{e}^{-\mu t_{1}}+\cos \left(\varphi_{0}+\varphi(\omega)+\omega t_{1}\right) \\
C\left(t_{1}, \varphi_{0}\right)= & -\frac{\omega_{0}^{2}}{\sigma} \sin \sigma t_{1} \mathrm{e}^{-\mu t_{1}} \\
D\left(t_{1}, \varphi_{0}\right)= & \left(\omega \cos \sigma t_{1} \sin \left(\varphi_{0}+\varphi(\omega)\right)+\frac{\sin \sigma t_{1}}{\sigma}\right. \\
& \left.\times\left(-\mu \omega \sin \left(\varphi_{0}+\varphi(\omega)\right)+\omega_{0}^{2} \cos \left(\varphi_{0}+\varphi(\omega)\right)\right)\right) \\
& \times \mathrm{e}^{-\mu t_{1}}-\omega \sin \left(\varphi_{0}+\varphi(\omega)+\omega t_{1}\right)
\end{aligned}
$$

The system (9) accepts non-trivial solutions for $\zeta_{0}$ only if the couple $\left(\phi_{0}, t_{1}\right)$ satisfies:

$A\left(t_{1}, \varphi_{0}\right) \times D\left(t_{1}, \varphi_{0}\right)-B\left(t_{1}, \varphi_{0}\right) \times C\left(t_{1}, \varphi_{0}\right)=0$

Which can be re-written as:

$$
\begin{aligned}
0= & -\sin \left(\varphi_{0}+\varphi(\omega)+\omega t_{1}\right) \\
& +2\left[\frac{\sin \sigma t_{1}}{\sigma} \cos \frac{\omega t_{1}}{2}\left(\begin{array}{c}
\frac{\omega_{0}^{2}}{\omega} \cos \left(\varphi_{0}+\varphi(\omega)+\frac{\omega t_{1}}{2}\right) \\
-\mu \sin \left(\varphi_{0}+\varphi(\omega)+\frac{\omega t_{1}}{2}\right)
\end{array}\right)\right. \\
& \left.-\cos \sigma t_{1} \sin \frac{\omega t_{1}}{2} \cos \left(\varphi_{0}+\varphi(\omega)+\frac{\omega t_{1}}{2}\right)\right] \mathrm{e}^{-\mu t_{1}} \\
& +\sin \left(\varphi_{0}+\varphi(\omega)\right) \mathrm{e}^{-2 \mu t_{1}}
\end{aligned}
$$


Whatever is the value of $\phi_{0}$, one can see that $t_{1}=0$ is always a trivial solution of the former equation. On another hand, letting $t_{1} \rightarrow+\infty$ asymptotically leads to the simplified Eq. (15)

$0=-\sin \left(\varphi_{0}+\varphi(\omega)+\omega t_{1}\right)$

whose solutions are $t_{1}=k(\pi / \omega)-\left(\varphi_{0}+\varphi(\omega)\right), k \in \mathbb{N}$. These long-term solutions corresponding to the established solution in forced motion are no more interesting in the present context where the focus is to be put on transients. Eq. (15) admits other short-term solutions, as can be seen in Fig. 3 where we have plotted together Eq. (15) and its asymptotic limit as a function of $t_{1}$. The most noticeable one is here for $t_{1} \simeq 5 s=\pi / \omega_{0}$. When $\mu$ is sufficiently small (weak damping), the first half-period of the motion roughly equals half the natural period of the oscillator. Now for a given parameter set $\left[\mu, \omega_{0}, \omega, F_{\text {ex }}\right]$, one can compute numerically the couples $\left(\phi_{0}, t_{1}\right)$ solution of (14) and then calculate the associated positions $\zeta_{0}$ given by:

$\zeta_{0}=-\frac{B\left(t_{1}, \varphi_{0}\right) F_{\mathrm{ex}} H(\omega)}{A\left(t_{1}, \varphi_{0}\right)}$

Finally, one has to select amongst all the couples $\left(\phi_{0}, t_{1}\right)$ solutions of (14) the couple maximizing $\zeta_{0}$. So it has been established how, when the body is locked in such a position $\zeta_{0 \max }$, releasing it when the phase of excitation force equals $\phi_{0}$ will drive it in position $-\zeta_{0 \max }$ with a velocity equal to 0 in $t_{1}$ seconds.

Working here under the assumption of harmonic forcing regime, this cycle will be reproduced periodically, alternating periods of motion and periods of rest. But it must be pointed out here that the period of this global response of the system to the harmonic forcing at frequency $\omega$ will not always equal the period of the excitation $T_{\mathrm{ex}}$, but can be integer multiple of it depending on the ratio $\omega / \omega_{0}$. Let $\omega_{0}=0.6, \mu=0.1$ and $F_{\text {ex }}=1$. We have computed $\zeta_{0 \max }$ for motion whose response period $T_{\text {out }}$ is either $T_{\text {ex }}$ either $3 T_{\text {ex }}$ or $5 T_{\text {ex }}$. In Fig. 4, we have plotted the three numerical

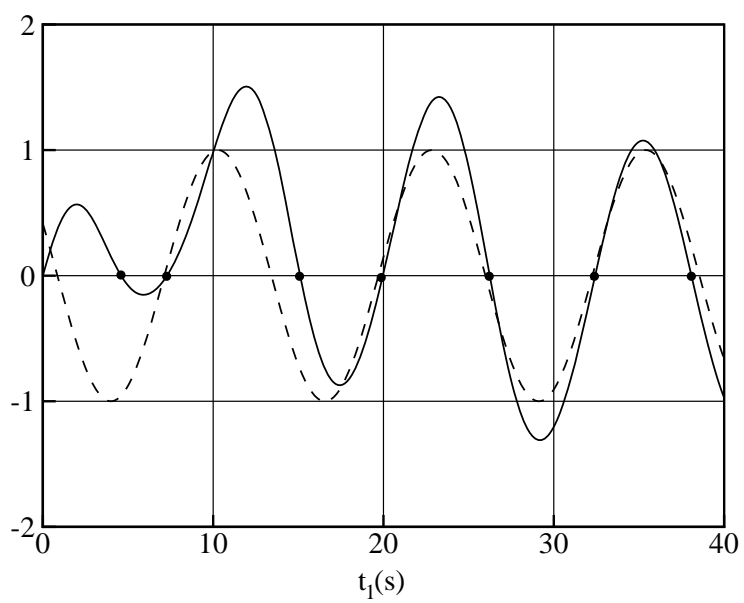

Fig. 3. Plot of Eq. (15) as a function of $t_{1}$ (solid) and asymptotic behaviour of Eq. (15) (dashed). Parameters are set equal to $\omega_{0}=0.6, \omega=0.5, \mu=0.05$.

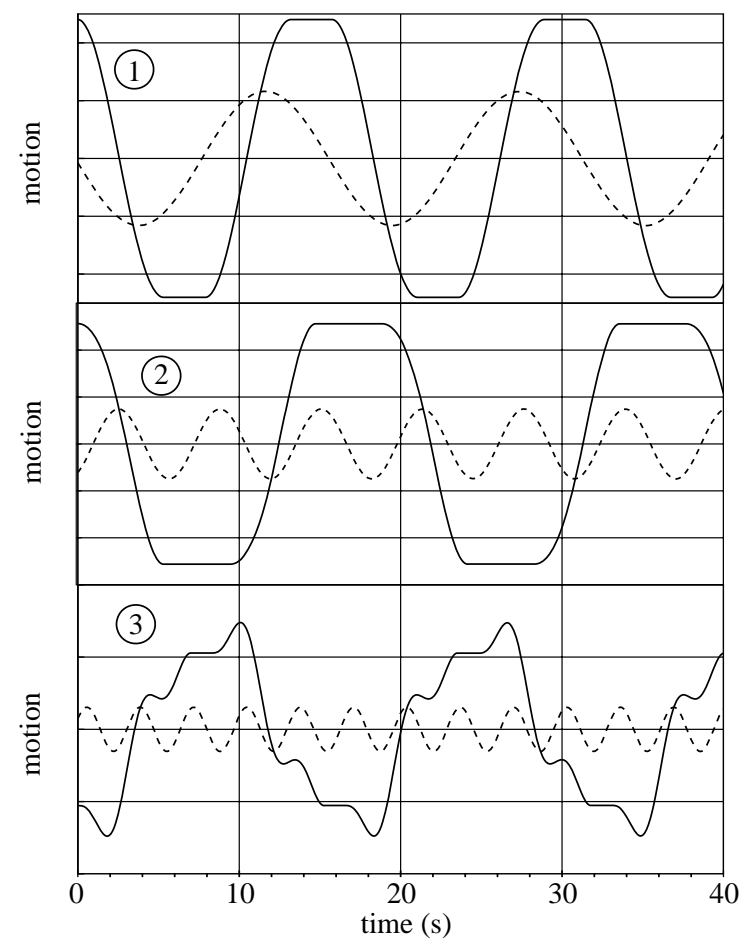

Fig. 4. Numerical simulations of the motion of a mechanical oscillator with and without latching control (alternated maxima ramps); $\omega_{0}=0.6, \mu=0.1$, $F_{\text {ex }}=1, \omega=1$.

simulations in time domain of the body response with and without latching control corresponding to the marks numbered one, two and three on Fig. 5. One can observe the huge magnification of the amplitude of the motion for the controlled system compared to the uncontrolled one. Rather than adapting the device parameters to the excitation force in order to optimize the linear body response, the latching control adapts the body response to the device and to the excitation in a nonlinear fashion. It is a kind of parametric resonance adaptation process as can be found in nonlinear oscillators theory. This exotic behavior was not observed in the previous studies which were, generally, made in the frequency domain approach which is based on the hypothesis that output frequency equals input frequency. Only time-domain simulations are able to highlight this kind of phenomenon.

Indeed, from Fig. 3 one can see that several solutions of (15) exist for $t_{1}$. The first one is located between 0 and $\left(T_{\mathrm{ex}} / 2\right)=(\pi / \omega)=6.28$ in this example, but we can see that in each segment $\left.[k+(1 / 2)) T_{\mathrm{ex}},(k+(3 / 2)) T_{\mathrm{ex}}\right], k \in \mathbb{N}$ there are two other values of $t_{1}$ solutions of (15). Now, selecting the value of $t_{1}$ between $\left.[k+(1 / 2)) T_{\mathrm{ex}},(k+(3 / 2)) T_{\mathrm{ex}}\right]$ leads to a period of the mass motion $T_{\text {out }}=2(k+(3 / 2)) T_{\text {ex }}$. Then, choosing $k=0$ results in a response period three times larger than the excitation period $\left(T_{\text {out }}=3 T_{\text {ex }}\right.$ ). (See Fig. $\left.4 \mathrm{~b}\right) ; k=1$ gives $T_{\text {out }}=5 T_{\text {ex }}$ (see Fig. $4 \mathrm{c}$ ), and so on.

The maximum amplitude $\zeta_{0 \max }$ of the controlled oscillator obtained by using the method explained above is plotted in Fig. 5 in a wide range of the frequency of 

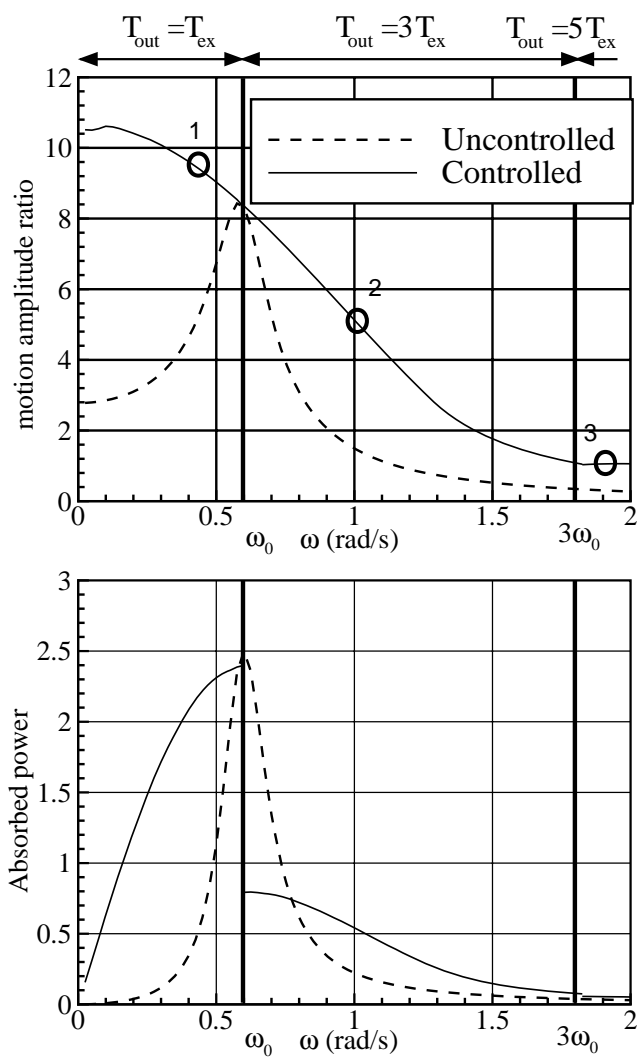

Fig. 5. Response amplitude and average absorbed power of a mechanical oscillator with and without latching control—alternated maxima ramps control strategy.

the excitation force, together with the transfer function of the uncontrolled oscillator, for the sake of comparison. In the upper Figure, the marks 1, 2, 3 correspond to the three different responses plotted in Fig. 4. In the lower figure, the average power absorbed by both the controlled and the uncontrolled system have been plotted.

First, one can appreciate the very large magnification of the body response brought by the discrete control when the wave frequency is off the natural frequency of the device. In the low frequency range (i.e. when $\omega<\omega_{0}$ ), it is a wellknown result which had been already observed and reported by M. Greenhow and S.P. White [17], among others. When the excitation frequency $\omega$ becomes higher than the natural frequency $\omega_{0}$, the period of the motion is no longer the period of the excitation, but three times this period (see Fig. $4-2$ ). Going further where $\omega>3 \omega_{0}$, the period of the response maximizing the amplitude is now five times the excitation period (see Fig. 4(3)); and so on. Considering now the mean absorbed power (Fig. 5), one can see that the latching control is highly beneficent when $\omega<\omega_{0}$, and that it is also interesting when $\omega>\omega_{0}$ except in a frequency band $(0.6<\omega \leqq 0.8)$ just above the natural frequency of the oscillator. In this zone, the gain in term of absorbed energy due to the amplification of the response amplitude is lower than the loss of absorbed energy during the latching of the body, in comparison with the uncontrolled motion. Indeed, in this zone, the latching duration equals approximately $3 /$ $2 T_{\mathrm{ex}}$. So, here, one would have better to leave the system uncontrolled, or to try another strategy as described in Section 3.3 to shorten this delay.

Let us now Fourier decompose the velocity of the controlled motion number 2 in Fig. 4(2) considering not $\omega$ but $\omega / 3$ as the fundamental frequency:

$\dot{\zeta}(t)=A_{0}+\sum_{n=1}^{+\infty}\left(A_{n} \cos \left(n \frac{\omega}{3} t\right)+B_{n} \sin \left(n \frac{\omega}{3} t\right)\right)$

Results are plotted in Fig. 6, where the term corresponding to the exciting frequency is represented by the second bar $(n=3)$. One can see here that the subharmonic mode $n=$ $1(\omega / 3)$ of the body response is far more energetic than the third one corresponding to frequency of the incident wave. No even mode are indeed present due to the antisymmetry of condition Eq. 8.

\subsection{Equal ending ramps}

The sub-harmonic phenomena described above are due, clearly, to the initial and final conditions $\zeta\left(t_{1}\right)=-\zeta(0)=$ $-\zeta_{0}$ which is one mean to impose global periodicity of the system response. This is not the unique way to achieve this goal, and another condition could be, indeed

$\zeta\left(t_{1}\right)=\zeta(0)=\zeta_{0}$

enforcing the system to return to its starting point at the end of the ramp.

The same algebra as developed in the above section can then be repeated after having changed only the final condition Eq. (18). This new condition results only in a change in the function $A\left(t_{1}, \phi_{0}\right)$ which now reads:

$A^{\prime}\left(t_{1}, \varphi_{0}\right)=\left(\cos \sigma t_{1}+\frac{\mu}{\sigma} \sin \sigma t_{1}\right) \mathrm{e}^{-\mu t_{1}}-1$

The same considerations and calculations as previously exposed finally results in typical system responses as plotted in Fig. 7. Again we can see that, for $\omega>\omega_{0}$, the optimal

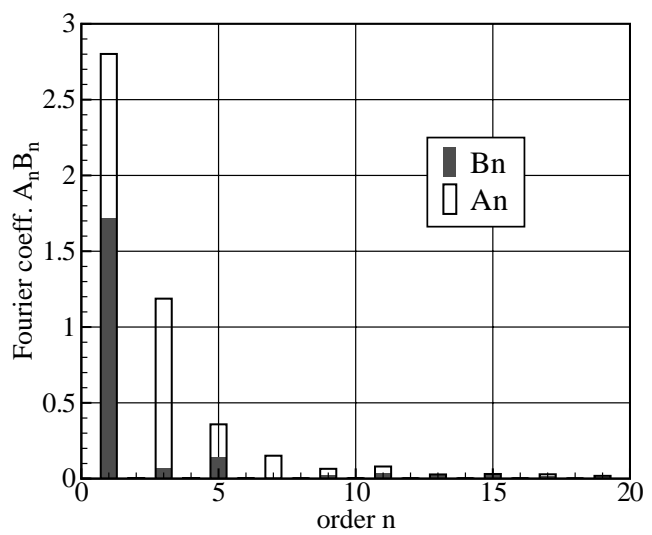

Fig. 6. Fourier coefficients of the velocity $\dot{\zeta}$ of the controlled motion of Fig. 4b. 


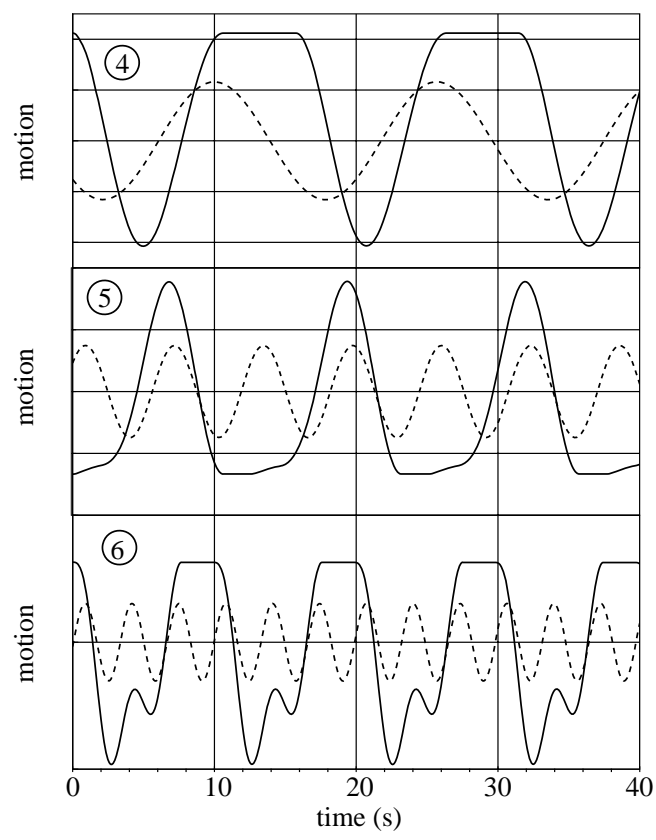

Fig. 7. Numerical simulations of the motion of a mechanical oscillator with and without latching control (equal ending ramps); $\omega_{0}=0.6, \mu=0.1$, $F_{\mathrm{ex}}=1, \omega=1$.
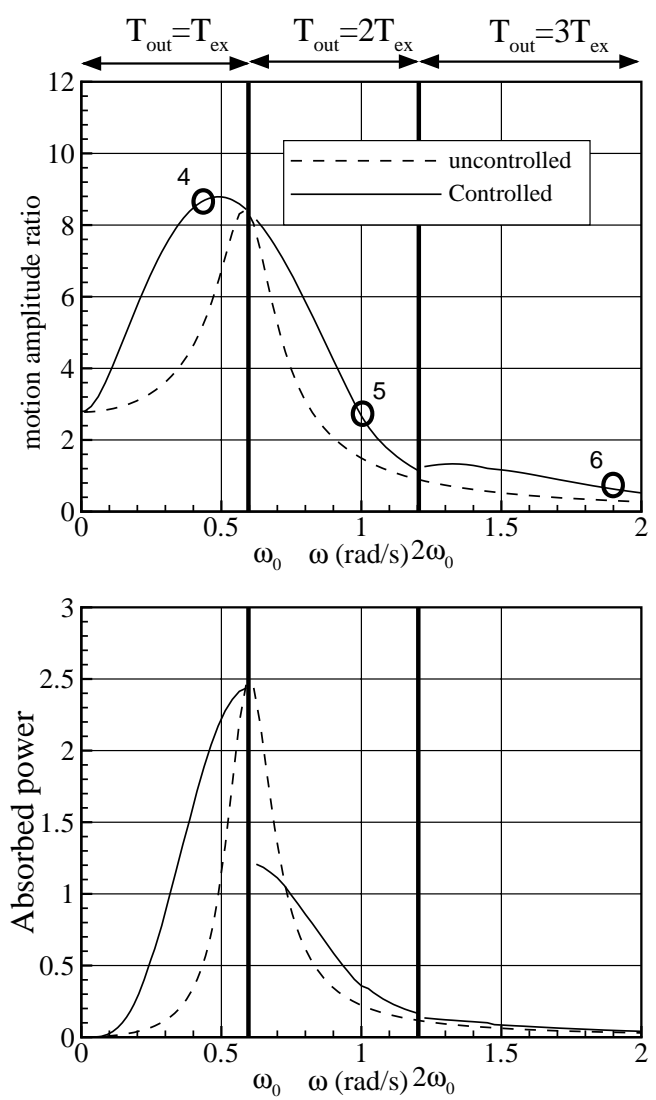

Fig. 8. Response amplitude and average absorbed power of a mechanical oscillator with and without latching control—equal ending ramps control strategy. response of the controlled system features sub-harmonic periods, multiples of the excitation period, but now beginning with period doubling, then tripling, etc...

The systematic computations reported above (Fig. 5) were performed again with this new latching condition; results are plotted in Fig. 8.

Again we can appreciate the response amplification on the top figure. Globally the amplification is less important than with the first strategy, particularly in the low frequency range $\omega<\omega_{0}$. The frequency band where latching control is inefficient $(0.6<\omega \leqq 0.75)$ is a bit narrower than in the previous case. Considering finally the bottom Fig. 8, we can see that the gain in term of absorbed power is globally lower with this latching strategy (equal ending ramps) than with the previous (alternated maxima ramps).

So we have now a better understanding on the best latching strategy for a basic oscillator in forced harmonic regime. Let us now consider the case of a wave activated energy converter, including the hydrodynamic terms of the motion equation, excited by regular and irregular waves.

\section{Application to a heaving wave energy converter in regular and in random waves}

\subsection{Governing equations}

We consider a wave energy converter in one mode of motion only, namely the heaving mode as described in Section 2. With the modelling adopted previously, the equation of motion is:

$\left(M+\mu_{\infty}\right) \ddot{\zeta}+\int_{0}^{t} \dot{\zeta}(\tau) K(t-\tau) \mathrm{d} \tau+B \dot{\zeta}+k \zeta=F_{\text {ex }}(t)$

With, according to Cummins (1962) [9]

$K(t)=\frac{2}{\pi} \int_{0}^{+\infty} C_{A}(\omega) \cos (\omega t) \mathrm{d} \omega$

where $C_{A}(\omega)$ is the hydrodynamical damping coefficient. Let us assume that $K$ can be approximated by a sum of exponentials:

$K(t) \simeq \sum_{i=1}^{N} \alpha_{i} \cdot \mathrm{e}^{\beta_{i} t}$

$K$ being real, the coefficients $\left(\alpha_{i}, \beta_{i}\right)$ are necessarily either real or associated by pair of complex conjugate. They can be determined by using, among others, Prony's method as described in [11] or in [7]. Let $I(t)$ be the convolution product to be evaluated:

$I(t)=\int_{0}^{t} \dot{\zeta}(\tau) K(t-\tau) \mathrm{d} \tau$ 
Using (21), this equation can be rewritten as a sum:

$$
I(t)=\sum_{i=1}^{N} I_{i}(t)
$$

With:

$I_{i}(t)=\int_{0}^{t} \dot{\zeta}(\tau) \alpha_{i} \mathrm{e}^{\beta_{i}(t-\tau)} \mathrm{d} \tau$

Differentiating $I_{i}$ leads to:

$$
\dot{I}_{i}(t)=\beta_{i} I_{i}(t)+\alpha_{i} \dot{\zeta}(t)
$$

With the initial conditions, according to (22):

$I_{i}(0)=0$

So the equation of motion (19) can be replaced by

$$
\left\{\begin{array}{l}
\left(M+\mu_{\infty}\right) \ddot{\zeta}(t)+\sum_{i=0}^{N} I_{i}(t)+B \dot{\zeta}(t)+k \zeta(t)=F_{\mathrm{ex}}(t) \\
\dot{I}_{i}(t)=\beta_{i} I_{i}(t)+\alpha_{i} \dot{\zeta}(t)
\end{array}\right.
$$

which indeed applies when the body is moving (during the ramps). Otherwise, when the body is latched, the equation of motion simplifies into:

$$
\left\{\begin{array}{l}
\dot{\zeta}(t)=0 \\
\dot{I}_{i}(t)=\beta_{i} I_{i}(t)
\end{array}\right.
$$

For random sea simulations, the excitation force was derived by discretizing the ITTC energy spectra [23] and using a random phase numerical generator

$S(\omega)=\frac{A}{\omega^{5}} \exp \left(\frac{-B}{\omega^{4}}\right)$

with:

$A=173 \frac{H_{1 / 3}^{2}}{T_{1}^{4}}$

$B=\frac{691}{T_{1}^{4}}$

In the reported applications, we have considered 300 frequencies between 0.01 and $6.0 \mathrm{rad} / \mathrm{s}$. Using our BEM seakeeping computational code AQUAPLUS, we have got the transfer function between the frequency of an incident harmonic wavetrain and the associated excitation force acting on the body. Then we were able to proceed to numerical simulation of motions by integration of the Eqs. (25) and (26) using a Runge-Kutta method. The body considered was a fixed volume fully submerged heaving cylinder, but there is no restrictions to apply the methods described here to any generic floating devices. The radius of the cylinder is $5 \mathrm{~m}$, the height is $10 \mathrm{~m}$ and it is submerged $10 \mathrm{~m}$ below the free surface. The following parameter set was used in the calculations: $M=360 \mathrm{t}, \quad \mu_{\infty}=155 \mathrm{t}, \quad k=240 \mathrm{kN} / \mathrm{m}, \quad B=C_{A}\left(\omega_{0}\right)=$ $27 \mathrm{kN} \mathrm{s} / \mathrm{m}$.
As in Section 3, we have considered here for simplification that the excitation force is known in the future of the current computation time, but keeping in mind that the final goal of our approach is to devise a causal control algorithm free of this constraint. Other optimal control approaches based on Pontragyin principle has been applied to this problem [19]; due to the fact that they rely on the knowledge of all the time range of the process, from the first to the last instant of running, they do not fit our final aim and we have preferred investigating another approach to the problem using some short-term prediction techniques such as Kalman extended filter [5], among others.

The aim of the present study being to compare three latching strategies, we have assumed herein that this shortterm prediction of the future excitation force is available every time during the computation. The implementation of this prediction algorithm in real world application is beyond the scope of the present paper.

\subsection{Three unlatching strategies}

The motion of a wave energy converter under latching control is a succession of time of rest and of transient motion, that we call ramps. The control variable is the release (or unlatching) time, or equivalently the latching duration. In [21], Korde calculated the optimal control law from a variational approach in the case of a wave energy converter where energy absorption and latching are achieved by means of pneumatic cylinders with open/ close valves. We have tried to apply such a method to our problem but the fact that the latching control is applied to the body, and not on the power take-off system, introduced a nonlinear constraint in the equation and we have not got, up to now, any interesting result using this method. We, therefore, decided to try a direct numerical approach.

Let us imagine that we are in a latched position at a time $t_{\mathrm{c}}$, with all the state variables being known. One can integrate Eq. (26) from $t_{\mathrm{c}}$ to $t_{\mathrm{c}}+t_{1}$, for any given latching duration, and then compute the motion the body would have for this $t_{1}$ during the next ramp by integrating Eq. (25), till the velocity vanishes again. The amount of energy absorbed during this ramp may be computed by integrating the product of the velocity by the PTO force over the time interval. In Fig. 9, we have plotted the energy absorbed during the next ramp when we vary the latching duration $t_{1}$. One can see the existence of successive local maxima and discontinuities in this curve. Discontinuities arise from the fact that we consider only the energy absorbed between two successive zeros of the velocity.

From these considerations, we have formulated three different strategies to determine the optimum unlatching time. These criteria are formulated in such a way that they can be applied in regular as well as irregular waves:

(1) The first control strategy we tested consists in choosing the latching time associated with the maximum of: 


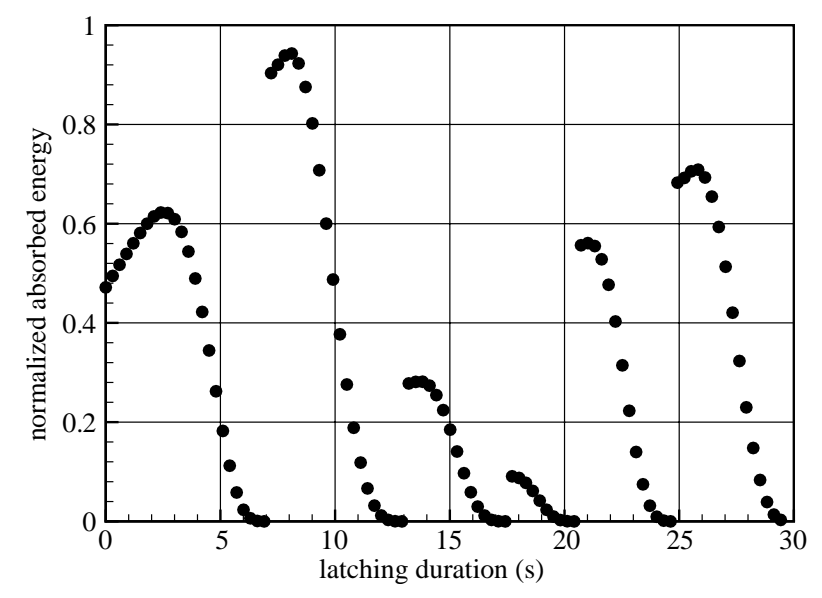

Fig. 9. Absorbed energy associated after current time $t$ as a function of latching duration in random incident wave.

- the absorbed energy for the first of the local maxima (e.g. $t_{l} \simeq 3 \mathrm{~s}$ in Fig. 9)

- the absorbed energy when latching time equals 0 otherwise.

In real implementation based on estimation of the exciting force as explained before, the error will indeed increase with the prediction duration. Moreover, on an engineering point of view, it is better to have more small oscillations than a few large ones. From these two practical arguments, we have chosen to select the first local maxima in the future (i.e. the first maximum), even if the second maximum is more powerful, as in Fig. 9.

(2) The second control strategy we have tested is quite the same as the first one, except that now we no longer maximize the absorbed energy but the amplitude of the foregoing ramp. We have plotted in Fig. 10 an example of a numerical simulation of the body motion in a real sea state under latching control according to this strategy number 2 . One can easily appreciate the amplifying effect of discrete control in random waves. On the lowest figure, we have plotted the accumulated energy by time integrating the instantaneous power. The mean absorbed power is, therefore, given by the mean slope of these curves. The benefit of latching is clearly exemplified by this example.

(3) The third strategy used is the same as the one used by Hals, Bjarte-Larsson and Falnes in [18]. By foreseeing at each time step the motion on the next ramp, we selected the right release time in order to put the next maximum of the velocity in phase with the next maximum of the excitation force. In regular monochromatic waves, this is an exact criterion of optimality for a WEC under continuous phase control. But in random waves where the phase becomes meaningless, this cannot be established theoretically. Nevertheless, we will assess the efficiency of this strategy after transposing it to discrete control in a real (random) waves context. This strategy will be numbered 3 in the sequel.

\section{Results in harmonic wave}

First, we compare the capacities of each proposed strategy in term of absorbed energy when the incident wave is monochromatic. We made time domain simulations of the body response for incident monochromatic wave with frequency in the range [0.1,2] $\mathrm{rad} / \mathrm{s}$, and with a common $1 \mathrm{~m}$ wave amplitude. Results are plotted in Fig. 11 in terms of relative capture width which is a common measure of point absorber devices efficiency in wave energy literature. This index is defined as the ratio of power absorbed over wave power incident in a width equal to the body diameter. For the sake of comparison we have also plotted on each graph the capture width for the uncontrolled system and the theoretical maximum (in linear theory). For such a single axisymmetric system in symmetric mode of motion, the maximum capture width equals to $\lambda /(2 \pi)[2,13,22]$. Note that it means that for low frequency waves, the absorbed power can possibly be greater than the incident power in the width of the body, implying capture widths greater than 1 . One can see that all three strategies greatly improve the system efficiency by enlarging considerably its bandwidth. The most efficient one in regular waves appears to be the second strategy (maximizing response amplitude) since it allows an improvement of the capture width whatever the frequency of the incident wave. Thus the result deduced previously from analytical calculations of optimal latching control applied to a mechanical oscillator seems to apply also to our hydrodynamical oscillator.

\section{Results in random sea}

Given a system and a latching strategy, the benefit that one can expect from such a control depends indeed on the spectral characteristics of the incident wave. Most commonly, a wave energy converter will work with wavetrain in which peak period $T_{1}$ is in the range $[6 s, 14 s]$. The characteristic height is also a variable, but, as long as we do not consider motion limitation by end stops, the latching control does not depend on the wave amplitude. Multiplying it by, say, $\alpha$, results in the multiplication by $\alpha$ for the body motion, since we work here in linearized theory, but the control points (i.e. the points where the body velocity vanishes) remain invariant, and so the moment of unlatching. We have made a great number of numerical simulations in random seas for different values of the peak period $T_{1}$ between 6 and $15 \mathrm{~s}$, repeating the 

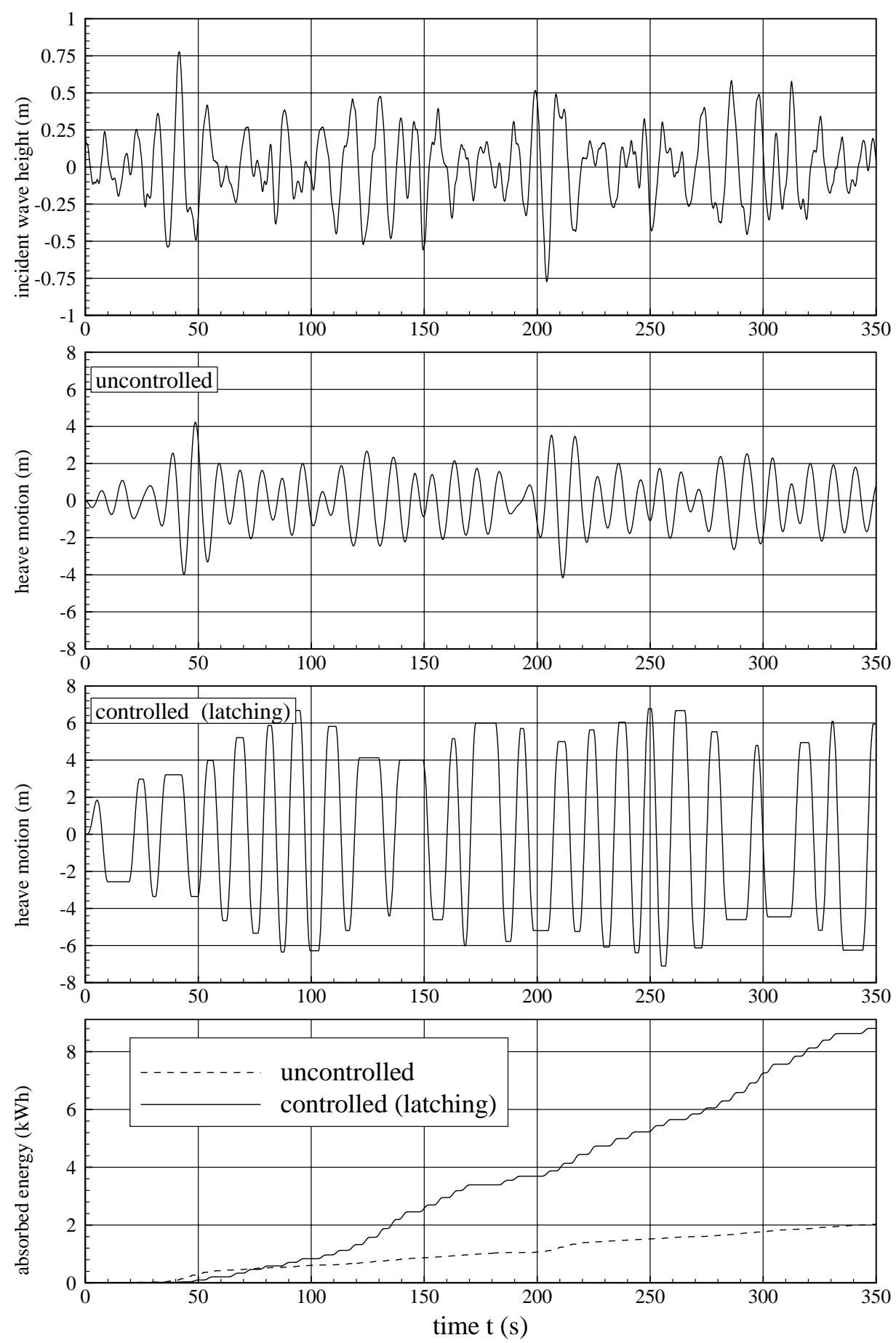

Fig. 10. Comparison of the motion with and without control using a strategy \#2 aiming at maximizing the body response amplitude. (a) Incident wave height, (b) free body response, (c) controlled body response and (d) accumulated energy in both cases.

same simulations for different random phase arrangements in order to obtain a mean estimation, for each one of the three control strategies. Results are plotted in terms of capture width in Fig. 12 as a function of the peak period $T_{1}$. For each strategy, we have also plotted, as a reference, the capture width we obtained for the same device without control. One can observe that the gain may be very important (up to a factor 4), in a range of peak period located around the natural period of the system $(10 \mathrm{~s})$, whereas the gain becomes negligible in the low period range. As for regular waves, the strategy number 2 based on maximizing the amplitude seems to give better results. For the free system, the most favorable range lies below the natural period of the device while the efficiency 

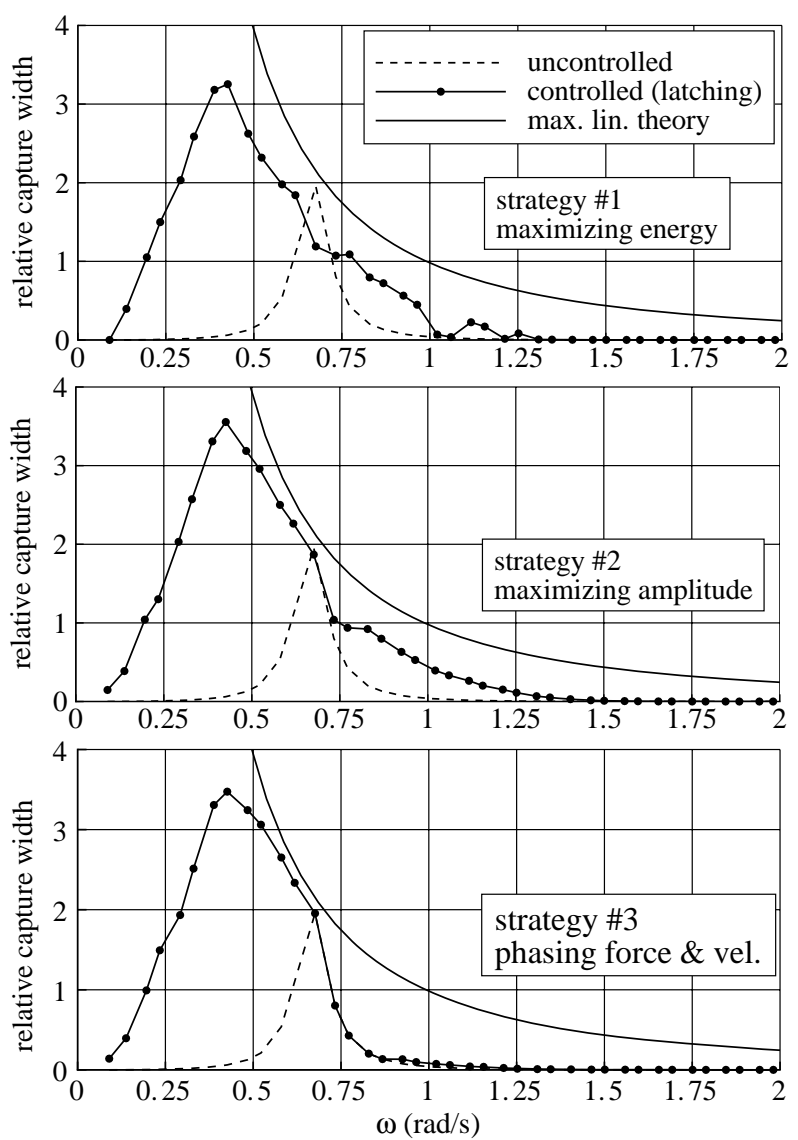

Fig. 11. Relative capture width of controlled and uncontrolled system in regular waves for the three control strategies tested.

remains very high for controlled devices in the long period range.

This is a key result of the study because it means that latching control will work with smaller devices (resulting in higher natural frequency), for a given power rating. This will be a helpful hint for engineers in charge of the design of such wave energy converters, for the placement of the natural period of the system with respect to the local sea state statistics at the working site.

As we pointed it out before, all these strategies will need to predict the excitation force in the close future or each unlatching event. In order to evaluate the size of the time window that we call 'close future', we define an half-period of the motion $T_{1 / 2}$ by the sum of the latching duration plus the duration of its associated ramp. The value of $T_{1 / 2}$ is the minimum of the prediction time required to achieve an efficient latching control by the method exposed herein. So we have plotted in Fig. 13, the mean $T_{\mathrm{m}}$ of all the computed $T_{1 / 2}$ as a function of the peak period of the incident wave train. We made three series of computations, keeping the same body geometry, but for three different values of the stiffness of the spring in order to obtain three different natural periods of the system: $T_{0} \in\{7 s, 10 s, 13 s\}$. One can see that $T_{\mathrm{m}}$ is
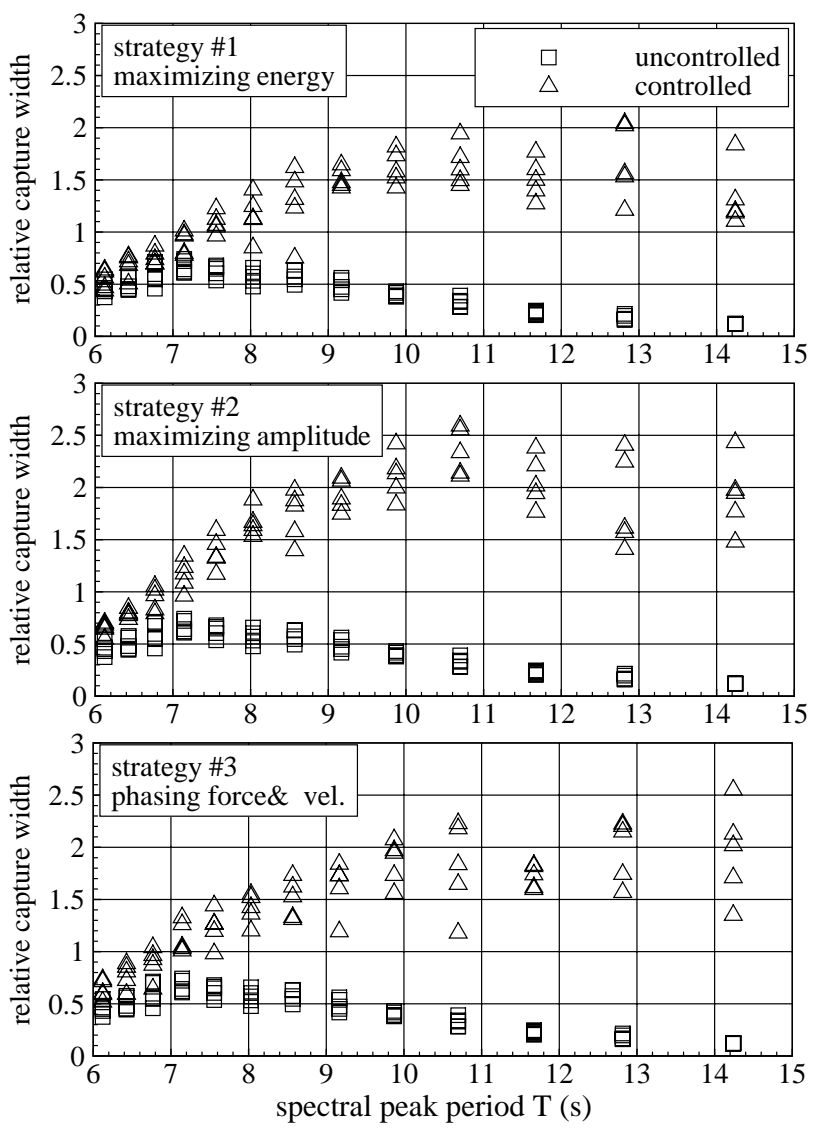

Fig. 12. Relative capture width of controlled and uncontrolled system in random sea.

minimum when the peak period equals the natural period. Moreover, when $T_{1}=T_{0}$, it seems that $T_{\mathrm{m}}=\left(T_{0} / 2\right)+2.0 \mathrm{~s}$. Anyway, $T_{\mathrm{m}}$ is always less than $T_{0}$, so we conclude that the length of the prediction time window is bounded by the natural period $T_{0}$ of the device.

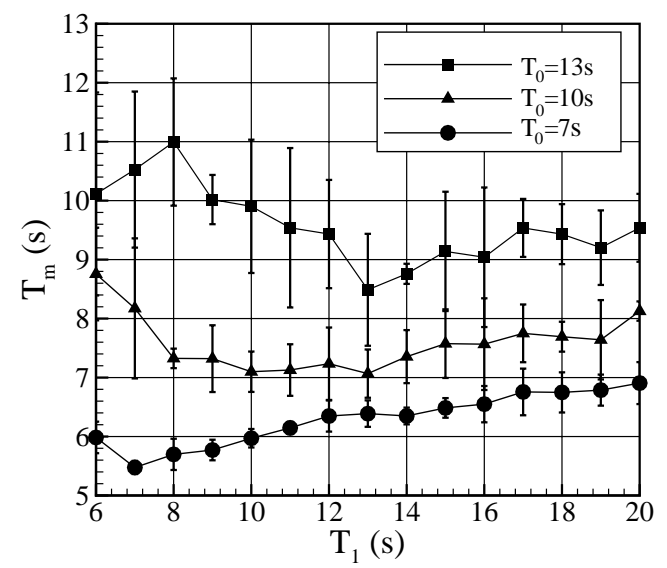

Fig. 13. Mean of the half-period of the controlled oscillator as a function of the peak period of the incident wave train for three different natural periods. 


\section{Conclusion}

By solving semi-analytically the equation of motion of a mechanical oscillator with a single degree of freedom, we have shown that latching control is able to increase significantly the amplitude of the motion, whatever the wave frequency. But the global behavior of the system, featuring period doubling, period tripling,..., of the response is much more like a time-varying system.

Thus, we compared a strategy aiming at the maximization of the amplitude of the motion of wave energy converter with a strategy aiming at the maximization of the absorbed energy and a strategy aiming at keeping velocity and excitation force in phase. Results in irregular waves show that, whatever is the control strategy, the efficiency of such a generic WEC device is considerably improved by latching control, provided one can predict the excitation force in the close future of the unlatching instant. In random waves, the control strategy (2) aiming at maximizing the amplitude of the motion gives approximately the same results as strategy (3) aiming at keeping velocity and excitation force in phase. In regular waves, the former one is the most efficient. Moreover, we found that the need for prediction of the future excitation force remains bounded by the natural period of the system. Further investigations should now be done to implement a method of short-term wave excitation forecasting in the latching control algorithm. It would permit the assessment of the feasibility of the present control strategy in real sea wave conditions.

\section{Acknowledgements}

The first and second authors are financially supported by the Centre National de la Recherche Scientifique, and the regional council of Pays de la Loire.

\section{References}

[1] www.wamit.com.

[2] Budal K, Falnes J. A resonant wave point absorber of ocean waves. Nature 1975;256:478-9.

[3] Budal K, Falnes J. Interacting point absorbers with controlled motion, in Power from Sea Waves. BM Count: Academic Press; 1980.
[4] Budal K, Falnes J, Iversen LC, Lillebeken PM, Oltedal G, Hals T, Onshus T, Hoy AS. The Norwegian wave-power buoy project. The second international symposium on wave energy utilization $1982 \mathrm{p}$. 323-44.

[5] Chatry G, Clément AH, Gouraud T. Self-adaptive control of a piston wave-absorber. Proc 8th Int Offshore Polar Eng Conf ISOPE98 1998; 1:127-33.

[6] Chatry G, Clément AH, Sarmento AJNA. Simulation of a selfadaptively controlled OWC in a nonlinear numerical wave tank. Int J Offshore Polar Eng 2000;10(4):269-75.

[7] Clément AH. Identification de la fonction de green de l'hydrodynamique transitoire par des modeles continus. Proc. 5emes Journees de l'Hydrodynamique 1995;319-32.

[8] Clément AH. Using differential properties of the green function in seakeeping computational codes. Proc 7th Int Conf Numer Ship Hydrol 1999;6(5):1-15.

[9] Cummins WE. The impulse response function and ship motions. Schiffstechnik 1962;491-502.

[10] Delhommeau G, Ferrant P, Guilbaud M. Calculation and measurement of forces on a high speed vehicle in forced pitch and heave. Appl Ocean Res 1992;14(2):119-26.

[11] Duclos G, Clément AH, Chatry G. Absorption of outgoing waves in a numerical wave tank using a self-adaptive boundary condition. Int J Offshore Polar Eng 2001;11(3):168-75.

[12] Eidsmoen H. Tight-moored amplitude-limited heaving buoy wave energy converter with phase control. Appl Ocean Res 1998;20:157-61.

[13] Evans DV. A theory for wave-power absorption by oscillating bodies. J Fluid Mech 1976;77:1-25.

[14] Falnes J. Ocean waves and oscillating systems, linear interactions including wave-energy extraction. 1st ed. Cambridge: Cambridge University Press; 2002.

[15] Falnes J. Optimum control of oscillation of wave-energy converters. Int J Offshore Polar Eng 2002;12(2):147-55.

[16] Falnes J, Budal K. Wave-power conversion by point absorbers. Norwegian Maritime Res 1978;6(4):2-11.

[17] Greenhow M, White SP. Optimal heave motion of some axisymmetric wave energy devices in sinusoidal waves. Appl Ocean Res 1997;19: $141-59$.

[18] Hals J, Bjarte-Larsson T, Falnes J. Optimum reactive control and control by latching of a wave-absorbing semisubmerged heaving sphere. Proc OMAE'02, 21st Int Conf Offshore Mech Arctic Eng 2002;1-9.

[19] Hoskin RE, Nichols NK. Optimal strategies for phase control of wave energy devices. In: Utilization of ocean waves: wave to energy conversion. 345 East 47th Street New York, New York 10017-2398: American Society of Civil Engineers; 1986. p. 184-99.

[20] Iversen LC. Numerical method for computing the power absorbed by a phase-controlled point absorber. Appl Ocean Res 1982;4(3): 173-80.

[21] Korde UA. Latching control of deep water wave energy devices using an active reference. Ocean Eng 2002;29(11):1343-55.

[22] Newman JN. The interaction of stationnary vessels with regular waves. Proc 11th Symp naval Hydrodyn 1976;491-501.

[23] Rawson KJ, Tupper FC.. 3rd ed Basic ship theory. vol. 1. London: Longman; 1983. 